\title{
Monitoring increased iron levels in multiple sclerosis using MRI
}
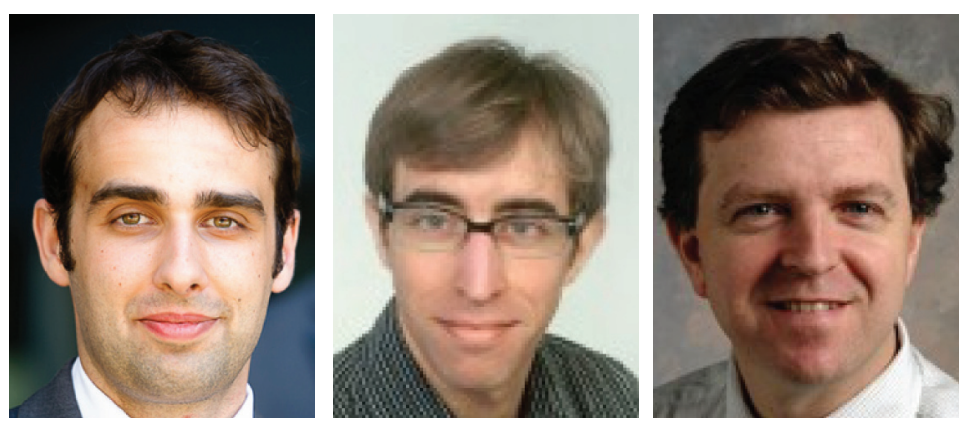

David A Rudko ${ }^{1,2}$, Juan M Racosta ${ }^{3}$ \& Marcelo Kremenchutzky ${ }^{*, 3}$

\section{Clinical symptoms \& pathophysiology of multiple sclerosis in relation to iron accumulation}

Multiple sclerosis (MS) is a chronic, inflammatory and neurodegenerative disease of the CNS characterized by a varied and unpredictable time course [1]. Though the specific cause is unknown, it appears to involve both genetic susceptibility and triggers such as a virus, dysregulated metabolism or environmental factors $[2,3]$. In most patients, MS consists of initial episodes of reversible neurological deficit, followed by progressive deterioration that can become severe and result in significant patient disability. In particular, evolution of the disease produces a spectrum of clinical features that may include motor weakness, sensory and gait disturbances, vision loss and cognitive changes [1].

Conventional MRI, using standard $\mathrm{T}_{1}$ and $\mathrm{T}_{2}$-weighted imaging has an important role in diagnosing MS [4]. However, it is now generally accepted that conventional MRI measures of MS disease status, such as single time point lesion load and lesion volume, have not correlated well with clinical symptoms or demonstrated significant predictive power for determining disease progression $[5,6]$. By contrast, post-mortem histopathological studies have consistently revealed free iron is present in brain tissue affected by MS [7]. Its presence makes it a potential candidate as an imaging biomarker. Not only are increased iron levels related to MS neurodegeneration, they also have a distinct signature on certain MRI contrasts [8-11].

Further support for the use of iron as a biomarker is provided by our understanding of the cellular-level, MS inflammatory response. The autoimmune targets in MS are believed to be cellular components of the CNS, normally inaccessible due to their location behind the blood-brain barrier. During disease initiation, entry of autoimmune cells ( $\mathrm{T}$ cells, B cells and monocytes) through the blood-brain barrier is triggered by cytokine and chemokine signals [2]. The inflammatory, autoimmune cells involved contain ferritin iron, an essential compound in normal cellular function. Because of the unique MRI signal produced by ferritin iron, MRI can be used to evaluate the cellular-level infiltrates that play a substantial role in the MS.

'Department of Physics \& Astronomy, Western University, London, ON, N6A 3K7, Canada

${ }^{2}$ Center for Functional \& Metabolic Mapping, Robarts Research Institute, London, ON, N6A 5K8, Canada

${ }^{3}$ Department of Clinical Neurological Sciences, Western University, London Health Sciences Centre, University Hospital, 339 Windermere Road, London, ON, N6A 5A5, Canada

*Author for correspondence: marcelo.kremenchutzky@lhsc.on.ca

\section{KEYWORDS}

- iron $\bullet \mathrm{MRI} \bullet$ multiple sclerosis

\section{"An integrative approach using}

MRI could ... lead to a better understanding of multiple sclerosis pathogenesis." 
"One particular area of the multiple sclerosis brain where iron accumulates more readily than others is the subcortical nuclei."
One particular area of the MS brain where iron accumulates more readily than others is the subcortical nuclei [11-14]. Elevated iron levels have been reported in subcortical nuclei in both clinically isolated syndrome (CIS) [12] and clinically definite MS patients [11,13]. Excessive levels of subcortical iron exert oxidative stress and result in cell death [15]. Consequently, monitoring the accumulation of iron in the subcortical nuclei and linking this to clinical disability is an emerging area of major interest in MRI of MS.

\section{MRI techniques for visualization of iron} in MS

Over the last two decades, several quantitative MRI techniques for monitoring iron in MS have been examined. These include: $R_{2}$ (spin echo transverse relaxation rate) - $[11,16,17]$ and $\mathrm{R}_{2}^{*}$ (gradient echo transverse relaxation rate)based relaxometry $[9,10]$; magnetic field correlation imaging (MFCI) [8]; and quantitative susceptibility mapping (QSM) $[12,13,18]$.

Because of its insensitivity to nonlocal background magnetic fields, $R_{2}$ has the potential to offer reliable and reproducible measurements of subcortical iron. At higher main magnetic fields ( $\geq 3 \mathrm{~T}$ ), subcortical $\mathrm{R}_{2}$ measurements may be particularly useful because the $R_{2}$ value of iron compounds increases linearly with main magnetic field strength [19]. Indeed, Lebel et al. [11] have demonstrated increased $\mathrm{R}_{2}$ in selected subcortical nuclei of RRMS patients compared with age and gender matched controls. In the pulvinar nucleus, increased $R_{2}$ values were correlated with extended disability status scale (EDSS) score. Recently, Walsh et al. [14] have also demonstrated a strong correlation between subcortical $\mathrm{R}_{2}$ in vivo and optical density of iron staining obtained from postmortem histopathology.

Currently however, whole-brain imaging is generally not performed with $\mathrm{R}_{2}$ mapping due to the associated longer acquisition times. If challenges related to the longer acquisition times and increased radiofrequency power deposition (particularly at higher main magnetic fields) can be addressed, this method will become more readily applicable for monitoring whole-brain iron in MS.

The use of $\mathrm{R}_{2}{ }^{*}$ as a biomarker of iron is attractive because of its relative ease and efficiency of acquisition using a standard multi-echo, gradient echo sequence. Khalil et al. have recently demonstrated in two separate studies $[9,10]$ that $\mathrm{R}_{2}{ }^{*}$ in subcortical nuclei is increased in MS and CIS patients compared with control subjects. Notably, subcortical $\mathrm{R}_{2}{ }^{*}$ levels were also increased in MS compared with both CIS and healthy controls. As well, using stepwise linear regression, subcortical $\mathrm{R}_{2}{ }^{*}$ values were correlated with age, disease duration, EDSS and mental processing speed.

These results are encouraging. However, care must be taken in the interpretation of $\mathrm{R}_{2}{ }^{*}$ values in brain tissue due to the significant impact of nontissue specific magnetic fields produced by susceptibility boundaries in the brain [20,21]. Additionally, interpretation of the $\mathrm{R}_{2}{ }^{*}$ signal becomes more challenging in cases where multiple tissue components (e.g., myelinated axons, microglia and oligodendrocytes) exist within an imaging voxel. In such a scenario, a simple mono-exponential decay model of $\mathrm{R}_{2}{ }^{*}$ is generally insufficient [22-24]. Future clinical studies using $\mathrm{R}_{2}{ }^{*}$ to monitor longitudinal iron accumulation in subcortical nuclei should help improve our understanding of the utility of this biomarker in predicting the MS progression. Further work should also examine the relationship between subcortical iron and more common imagingbased measures of MS such as lesion load and brain atrophy. Investigating these relationships has the potential to more clearly define consistent, whole-brain imaging biomarkers of MS.

Compared to $\mathrm{R}_{2}$ and $\mathrm{R}_{2}{ }^{*}$ mapping, MFCI advantageously removes the effect of dipole-dipole interactions and macroscopic, inhomogeneous magnetic fields which can make quantitation more difficult [25,26]. Moreover, because the MFC parameter scales quadratically with main magnetic field strength $[25,26]$, its acquisition using high-field MRI is an attractive option. MFC values generally also have a larger dynamic range compared with $R_{2}$ and $\mathrm{R}_{2}{ }^{*}$ values $[25,26]$. As a result, this method may be useful for mapping subtle differences in iron concentration which are not detected using other iron imaging techniques. Ge et al. [8] have demonstrated significant increases in MFC of RRMS patients compared with matched control subjects in the globus pallidus, putamen and thalamus. The increases in the thalamus and globus pallidus were associated with white matter (WM) lesion load. Moderate correlations were also found between MFC in the thalamus and neuropsychological test scores [8].

To date, however there have been few MFCI studies of MS due to the more complicated pulse sequence used with its acquisition (an asymmetric spin-echo sequence with echo-planar imaging 
readout). Future research, focusing on larger MS patient cohorts with varying disease subtypes, is necessary to more fully understand the utility of this method. A comparison of MFCIderived iron measures to those obtained from relaxometry and QSM would also be instructive.

QSM is a relatively new, MRI-based, iron mapping method that has shown an increased sensitivity to changes in subcortical iron associated with normal ageing [27] and MS diseaserelated processes [13]. The increased sensitivity has been hypothesized to result from the ability of QSM to measure the mean magnetic field perturbation in an imaging voxel, as opposed to the signal relaxation of protons in water molecules [28]. The mean magnetic field perturbation can be more directly linked to the amount of iron in tissue [27]. Additionally, QS maps can be efficiently derived from standard gradient echo imaging data. For example, Langkammer et al. [13] have demonstrated elevated QS values in subcortical nuclei of CIS and clinically definite MS patients compared with controls. Subcortical nuclei QSM values were higher with increasing EDSS and lower with both normalized total volume of gray matter and cortical gray matter volume [13].

Nevertheless, the use of QSM is still hampered by challenges related to proper processing of the MRI phase information [29-31]. Equally relevant, the specific tissue structures and chemical compounds which contribute to the QS signal within a voxel are not completely understood. With more advanced biophysical models and improved QS map calculation, however, QSM has the potential to serve a significant role in the efficient detection of whole-brain distributions of iron in MS.

On balance, although iron-sensitive MRI has already shown potential as a clinical biomarker of MS, its exact utility for predicting onset, tracking disease progression or monitoring therapy is yet to be determined. Present iron imaging techniques offer complementary information to the standard scans conventionally used in MS diagnosis. With additional refinement, they have the potential to assume a more substantial role in both tracking MS progression and stratifying MS disease subtypes.

\section{Clinical \& diagnostic significance of tracking iron accumulation using MRI}

Regardless of whether increased iron levels are a cause or a consequence of the MS pathological process, their detection may, in future, be used to measure the effects of treatment and predict disease progression. Several candidate mechanisms have been proposed for the role of iron in MS. The most general of these posits that, when iron becomes unchelated or is metabolically dysregulated, cell damage occurs due to the catalysis of free radical oxidations induced by free iron species [15,32]. In particular, lipid peroxidation reactions catalyzed by iron are believed to be one of the key initiators of cell death in MS [15]. In these reactions, the unsaturated lipids in cell membranes react with free radical oxygen species to produce toxic lipid hydroperoxides, which contribute to DNA damage and result in cell death [15].

Current understanding of the underlying cellular sources of iron-induced tissue damage has led to research investigating iron chelators and biochemical inhibitors of lipid peroxidation for MS therapy [33-35]. Although these therapies have demonstrated effectiveness in animal models [36,37], in clinical trials with progressive MS patients they have failed to demonstrate significant improvement in patient symptoms [38,39].

From a diagnostic standpoint, longitudinal imaging studies of iron may allow clinicians to pre-empt worsening clinical symptoms by knowing when to administer therapy. QSM has already recognized there is a progressive increase in iron levels in newly formed, contrast-enhancing WM lesions [18]. This has been attributed to iron-bearing cell infiltration [18]. As well, nonenhancing WM lesions have characteristically shown an accentuated iron and ferritin rim with a reduction in iron at the lesion core [40]. Such MRI signatures are believed to signify an acute inflammatory and demyelinating MS response. Administering therapy catered to the inflammatory phases of the MS response could, accordingly, be facilitated by using iron-sensitive MRI. However, for this to be practical, the cost-effectiveness and specificity of iron-imaging methods need to be further addressed.

Considering iron accumulation in separate brain regions (e.g., WM lesions, subcortical nuclei and normal-appearing WM) has been a major focus of current MRI research in MS. Future studies should examine the relationship between iron accumulations in different structures across the whole brain. This would offer an improved depiction of the full disease process and help clinicians understand which parts of

\section{"Future studies should examine the relationship between iron accumulations in different structures across the whole brain.”}


the brain are most significantly affected in the various MS subtypes.

\section{Conclusion}

Is iron solely a byproduct of the MS inflammatory process? Or does it play a causal role in the progressive demyelination and axonal loss? Is increased iron storage a function of oligondendrocyte impairment and subsequent iron release? These questions remain unanswered. An integrative approach using MRI could help answer the above questions and lead to a better understanding of MS pathogenesis. In doing so, it could also provide increased insight into the efficacy of such novel MS therapies as iron chelators and inhibitors of iron-related oxidative stress.

\section{Financial \& competing interests disclosure}

The authors have no relevant affiliations or financial involvement with any organization or entity with a financial interest in or financial conflict with the subject matter or materials discussed in the manuscript. This includes employment, consultancies, honoraria, stock ownership or options, expert testimony, grants or patents received or pending or royalties.

No writing assistance was utilized in the production of this manuscript.

\section{References}

1 Kremenchutzky M, Rice GP, Baskerville J, Wingerchuk DM, Ebers GC. The natural history of multiple sclerosis: a geographically based study 9: observations on the progressive phase of the disease. Brain 129(3), 584-594 (2006).

2 Ontaneda D, Hyland M, Cohen JA. Multiple sclerosis: new insights in pathogenesis and novel therapeutics. Annu. Rev. Med. 63, 389-404 (2012).

3 Sospedra M, Martin R. Immunology of multiple sclerosis. Annu. Rev. Immunol. 23, 683-747 (2005).

4 Polman CH, Reingold SC, Banwell B et al. Diagnostic criteria for multiple sclerosis: 2010 revisions to the McDonald criteria. Ann. Neurol. 69, 292-302 (2011).

5 Filippi M, Rocca MA, De Stefano N et al. Magnetic resonance techniques in multiple sclerosis: the present and the future. Arch. Neurol. 68, 1514-1520 (2011).

6 Ceccarelli A, Bakshi R, Neema M. MRI in multiple sclerosis: a review of the current literature. Curr. Opin. Neurol. 25, 402-409 (2012)

7 Yao B, Bagnato F, Matsuura E et al. Chronic multiple sclerosis lesions: characterization with high-field-strength MR imaging. Radiology 262, 206-215 (2012).

8 Ge Y, Jensen JH, Lu H et al. Quantitative assessment of iron accumulation in the deep gray matter of multiple sclerosis by magnetic field correlation imaging. AJNR Am. J. Neuroradiol. 28, 1639-1644 (2007).

9 Khalil M, Enzinger C, Langkammer C et al. Quantitative assessment of brain iron by R2* relaxometry in patients with clinically isolated syndrome and relapsing-remitting multiple sclerosis. Mult. Scler. 15, 1048-1054 (2009).
10 Khalil M, Langkammer C, Ropele S et al. Determinants of brain iron in multiple sclerosis: a quantitative 3T MRI study. Neurology 77, 1691-1697 (2011).

11 Lebel RM, Eissa A, Seres P, Blevins G, Wilman AH. Quantitative high-field imaging of sub-cortical gray matter in multiple sclerosis. Mult. Scler. 18, 433-441 (2012).

12 Al-Radaideh AM, Wharton SJ, Lim SY et al. Increased iron accumulation occurs in the earliest stages of demyelinating disease: an ultra-high field susceptibility mapping study in clinically isolated syndrome. Mult. Scler. 19, 896-903 (2013).

13 Langkammer C, Liu T, Khalil M et al. Quantitative susceptibility mapping in multiple sclerosis. Radiology 267, 551-559 (2013).

14 Walsh AA, Lebel RM, Eissa A et al. Multiple sclerosis: validation of MR imaging for quantification and detection of iron. Radiology 267, 531-542 (2013).

15 Nunez MM, Urrutia P, Mena N, Aguirre P, Tapia V, Salazar J. Iron toxicity in neurodegeneration. Biometals 25, 761-776 (2012).

16 Laule C, Leung E, Lis DK et al. Myelin water imaging in multiple sclerosis: quantitative correlations with histopathology. Mult. Scler. 12, 747-753 (2006).

17 Laule C, Kozlowski P, Leung E, Li DK, Mackay AL, Moore GR. Myelin water imaging of multiple sclerosis at $7 \mathrm{~T}$ : correlations with histopathology. Neuroimage 40, 1575-1580 (2008).

18 Chen W, Gauthier SA, Gupta A et al. Quantitative susceptibility mapping of multiple sclerosis lesions at various ages. Radiology 271, 183-192 (2014).

19 Gelman N, Gorell JM, Barker PB et al. MR imaging of human brain at $3.0 \mathrm{~T}$ : preliminary report on transverse relaxation rates and relation to estimated iron content. Radiology 210, 759-767 (1999).

20 Fernandez-Seara MA, Wehrli FW. Postprocessing technique to correct for background gradients in image-based $\mathrm{R}^{*}(2)$ measurements. Magn. Reson. Med. 44, 358-366 (2000).

21 Peters AA, Brookes MJ, Hoogenraad FG et al. T2* measurements in human brain at 1.5, 3 and 7 T. Magn. Reson. Imaging 25, 748-753 (2007).

22 Du YY, Chu R, Hwang D et al. Fast multislice mapping of the myelin water fraction using multicompartment analysis of T2* decay at 3T: a preliminary postmortem study. Magn. Reson. Med. 58, 865-870 (2007).

23 Hwang D, Kim DH, Du YP. In vivo multi-slice mapping of myelin water content using T2* decay. Neuroimage 52, 198-204 (2010).

24 Kwon OI, Woo EJ, Du YP, Hwang D. A tissue-relaxation-dependent neighboring method for robust mapping of the myelin water fraction. Neuroimage 74, 12-21 (2013).

25 Jensen JJ, Chandra R, Ramani A et al. Magnetic field correlation imaging. Magn. Reson. Med. 55, 1350-1361 (2006).

26 Jensen JJ, Szulc K, Hu C et al. Magnetic field correlation as a measure of iron-generated magnetic field inhomogeneities in the brain. Magn. Reson. Med. 61, 481-485 (2009).

27 Bilgic B, Pfefferbaum A, Rohlfing T, Sullivan EV, Adalsteinsson E. MRI estimates of brain iron concentration in normal aging using quantitative susceptibility mapping. Neuroimage 59, 2625-2635 (2012).

28 Deistung A, Schafer A, Schweser F, Biedermann U, Turner R, Reichenbach JR. Toward in vivo histology: a comparison of quantitative susceptibility mapping (QSM) 
with magnitude-, phase-, and R2*-imaging at ultra-high magnetic field strength.

Neuroimage 65, 299-314 (2013).

Schweser F, Deistung A, Lehr BW,

Reichenbach JR. Quantitative imaging of intrinsic magnetic tissue properties using MRI signal phase: an approach to in vivo brain iron metabolism? Neuroimage 54, 2789-2807 (2011).

30 Wharton S, Schäfer A, Bowtell R. Susceptibility mapping in the human brain using threshold-based k-space division. Magn. Reson. Med. 63, 1292-1304 (2010).

Wharton S, Bowtell R. Whole-brain susceptibility mapping at high field: a comparison of multiple-and single-orientation methods. Neuroimage 53, 515-525 (2010).

LeVine SS. Iron deposits in multiple sclerosis and Alzheimer's disease brains. Brain Res. 760, 298-303 (1997).
33 LeVine SS, Chakrabarty A. The role of iron in the pathogenesis of experimental allergic encephalomyelitis and multiple sclerosis. Ann. NY Acad. Sci. 1012, 252-266 (2004).

34 Richardson DD. Novel chelators for central nervous system disorders that involve alterations in the metabolism of iron and other metal ions. Ann. NY Acad. Sci. 1012, 326-341 (2004).

35 Whitnall M, Richardson DR. Iron: a new target for pharmacological intervention in neurodegenerative diseases. Semin. Pediatr. Neurol. 13, 186-197 (2006).

36 Pedchenko TT, LeVine SM.

Desferrioxamine suppresses experimental allergic encephalomyelitis induced by MBP in SJL mice. J. Neuroimmunol. 84, 188-197 (1998).

37 Mitchell KK, Dotson AL, Cool KM, Chakrabarty A, Benedict SH, LeVine SM.
Deferiprone, an orally deliverable iron chelator, ameliorates experimental autoimmune encephalomyelitis. Mult. Scler. 13, 1118-1126 (2007).

38 Lynch SS, Peters K, LeVine SM. Desferrioxamine in chronic progressive multiple sclerosis: a pilot study. Mult. Scler. 2, 157-160 (1996).

39 Lynch SS, Fonseca T, LeVine SM. A multiple course trial of desferrioxamine in chronic progressive multiple sclerosis. Cell. Mol. Biol. (Noisy-le-grand) 46, 865-869 (2000).

40 Bagnato F, Hametner S, Yao B et al. Tracking iron in multiple sclerosis: a combined imaging and histopathological study at 7 Tesla. Brain 134, 3602-3615 (2011). 